\title{
Landespflegekammer formuliert Forderungen an Politik und Arbeitgeber
}

\section{Hier steht eine Anzeige.}

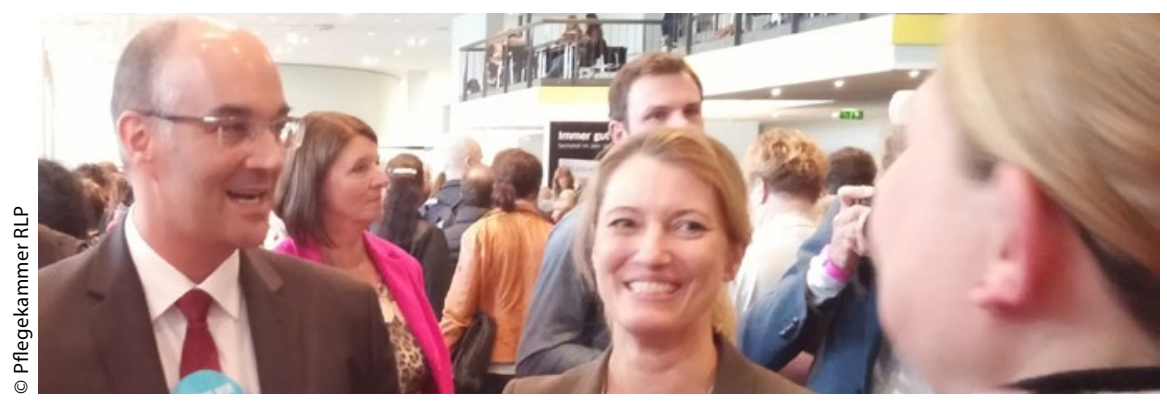

Kammerpräsident Dr. Markus Mai auf dem 1. Pflegetag Rheinland-Pfalz

— Mit rund 1.200 Besuchern und über 50 Ausstellern fand am 3. Mai 2017 der erste Pflegetag Rheinland-Pfalz in Mainz statt. Die rheinland-pfälzische Ministerpräsidentin Malu Dreyer hob in ihrem Grußwort die gesamtgesellschaftliche Bedeutung beruflich Pflegender hervor und bekräftigte die Notwendigkeit einer institutionellen Interessenvertretung in Form einer Pflegekammer. Sie betonte, dass sich die Landesregierung mit ihrer Pflegepolitik für die Einführung einer generalistischen Pflegeausbildung einsetze, die den Bedarfen der Zukunft gerecht werde.

Die Landespflegekammer Rheinland-Pfalz als Veranstalter legte den Fokus des Pflege- tags auf pflegefachliche Themen und regionale Projekte. Auf einer "Kammermeile" informierte sie die Besucher über ihre Arbeit. Gleichwohl nutzten Kammerpräsident Dr. Markus Mai und Kammervizepräsidentin Sandra Postel im Vorfeld des Pflegetags die Gelegenheit, ihre Forderungen an Politik und Arbeitgeber zu formulieren. Auf der Agenda stehen dabei drei zentrale Themen: Krankenhausinvestitionen der Landesregierung, die Personalbemessung, Arbeitsbedingungen und tariflichen Verbesserungen in der Altenpflege.

(sk)

www.pflegekammer-rlp.de

Nachhaltigkeit in der Pflege

\section{DBfK verabschiedet Resolution zu UN-Entwicklungszielen}

- Der Bundesvorstand und die Delegierten des Deutschen Berufsverbands für Pflegeberufe (DBfK) verabschiedeten am diesjährigen Internationalen Tag der Pflege (12. Mai) eine Resolution für Pflegende. Darin erklärten sie, sich für das Erreichen der UN-Entwicklungsziele einzusetzen. Die Resolution greift acht ausgewählte Ziele auf, die unmittelbar mit der Tätigkeit von professionell Pflegenden verknüpft sind: keine Armut, kein Hunger, Gesundheit und Wohlergehen, hochwertige Bildung, Geschlechtergleichheit, Menschenwürdige Arbeit und Wirtschaftswachstum, nachhaltige Städte und Gemeinden sowie Frieden, Gerechtigkeit und starke Institutionen.
DBfK-Präsidentin Prof. Christel Bienstein erklärte, dass die nachhaltigen Entwicklungsziele in Deutschland ohne maßgebliche Einbindung der Pflegeberufe auf allen politischen Ebenen nicht zu erreichen seien. Dabei sei nationales politisches Handeln an diesen Zielen auszurichten und müsse sich letztlich auch daran messen lassen.

Das Papier mündet in einen Katalog von zehn Kernforderungen, zu denen unter anderem mehr Mitspracherechte, bessere Arbeitsbedingungen, höhere Personalbemessung, Durchsetzung der Arbeitsschutzgesetze und der Wille zur Veränderung gehören.

www.dbfk.de 